\title{
THE WIDTH OF VERBAL SUBGROUPS IN THE GROUP OF UNITRIANGULAR MATRICES OVER A FIELD
}

\author{
Agnieszka Bier \\ e-mail: agnieszka.bier@polsl.pl
}

\begin{abstract}
Let $K$ be a field and let $U T_{n}(K)$ and $T_{n}(K)$ denote the groups of all unitriangular and triangular matrices over field $K$, respectively. In the paper, the lattices of verbal subgroups of these groups are characterized. Consequently the equalities between certain verbal subgroups and their verbal width are determined. The considerations bring a series of verbal subgroups with exactly known finite width equal to 2 . An analogous characterization and results for the groups of infinitely dimensional triangular and unitriangular matrices are established in the last part of the paper.
\end{abstract}

\section{INTRODUCTION \& STATEMENT OF RESULTS.}

Let $K$ be a field. We consider the subgroups $U T_{n}(K), T_{n}(K)$ and $D_{n}(K)$ of the general linear group $G L_{n}(K), n \in \mathbf{N}$. $U T_{n}(K)$ is a group consisting of all upper triangular matrices of size $n \times n$ with unity entries on the main diagonal:

$$
U T_{n}(K)=\left\{A \in G L_{n}(K) \mid A=\mathbf{1}_{\mathbf{n}}+\sum_{1 \leq i<j \leq n} a_{i, j} e_{i, j}, \quad a_{i, j} \in K\right\},
$$

where $\mathbf{1}_{\mathbf{n}}$ denotes the unity matrix of size $n \times n$ and $e_{i, j}$ denotes the matrix with unity in the place $(i, j)$ and zeros elsewhere.

$U T_{n}(K)$ is a normal subgroup in the group $T_{n}(K)$ of all invertible upper triangular matrices of size $n \times n$. Moreover, $T_{n}(K)$ can be represented as a semidirect product:

$$
T_{n}(K)=D_{n}(K) \curlywedge U T_{n}(K),
$$

where $D_{n}(K)$ is the group of all invertible diagonal matrices of size $n \times n$. In [9] A. Weir determined the characteristic subgroups in $U T_{n}(K)$ over fields of odd prime characteristic. A more general description of characteristic subgroups of $U T_{n}(K)$ for an arbitrary field $K,|K|>2$, was given later by Levchuk in [5]

MSC 20E15, 20F14, 20F18, 20G15 
and [6]. The case of a 2-element field $(|K|=2)$ was however not covered in any of the cited results.

Let $W$ be a set of group words over an alphabet $X=\left\{x_{1}, x_{2}, \ldots\right\}$. For an arbitrary group $G$ the verbal subgroup of group $G$ with respect to the set $W$ is a group $V_{W}(G)$ generated by all values of words from $W$ in group $G$. If $W=\{w\}$ then we write $V_{w}(G)$ instead of $V_{W}(G)$. In a nilpotent group $G$ every verbal subgroup is generated by a single word, i.e. for every set of words $W$ there exists a word $w$ such that $V_{W}(G)=V_{w}(G)$.

The width wid ${ }_{W}(G)$ of the verbal subgroup $V_{W}(G)$ is the smallest (if such exists) number $l \in \mathbf{N}$ such that every element $g \in V_{W}(G)$ can be represented as a product of $l$ values of words from $W$ in group $G$. If such number does not exist, we say that $w_{i} d_{W}(G)=\infty$. One should pay attention to the fact that the same verbal subgroup considered with different generating sets of words may have different verbal width.

Every verbal subgroup is a fully characteristic subgroup. The converse statement is true in some groups, for example in the free group, however in general this is false. In this paper we will give another example of groups in which fully characteristic subgroups coincide with verbal subgroups.

We start the considerations with introducing some special types of words and the resulting verbal subgroups. In every group $G$ the words:

$$
c_{1}=x_{1}, \quad c_{i+1}=\left[c_{i}\left(x_{1}, \ldots, x_{i}\right), x_{i+1}\right], \quad i=1,2, \ldots,
$$

called the left-normed basic commutators, generate a series of verbal subgroups $V_{c_{i}}(G)$. The subgroups $V_{c_{i}}(G)$ constitute the lower central series of $G$ :

$$
G=\gamma_{1}(G) \geq \gamma_{2}(G) \geq \ldots,
$$

in which $\gamma_{i}(G)=V_{c_{i}}(G)$.

Next, for $n \in \mathbf{N}$ we define the words $d_{n}$ as follows:

$d_{1}\left(x_{1}\right)=x_{1}, \quad d_{n+1}\left(x_{1}, x_{2}, \ldots, x_{2^{n}}\right)=\left[d_{n}\left(x_{1}, x_{2}, \ldots, x_{2^{n-1}}\right), d_{n}\left(x_{2^{n-1}+1}, \ldots, x_{2^{n}}\right)\right]$.

The verbal subgroups $V_{d_{i}}(G)$ constitute the derived series of group $G$ :

$$
G \leq G^{\prime} \leq G^{\prime \prime} \leq \ldots
$$

The two types of words introduced above are the examples of a wider class of words, namely the outer-commutator words. An outer commutator word of weight $n$ is defined recursively as follows. The outer-commutator word of weight 1 is the single-letter word of the form $\omega_{1}=x_{i}$, where $x_{i} \in X$. Then, if $u_{r}=u\left(x_{i_{1}}, \ldots, x_{i_{r}}\right)$ and $v_{n-r}=v\left(x_{i_{r+1}}, \ldots, x_{i_{n}}\right)$, with $x_{i_{j}} \in X, 1 \leq j \leq n$ are outer-commutator words of weight $r$ and $n-r$ respectively, such that $x_{i_{j}} \neq x_{i_{k}}$ for $j \neq k$, then the word $\left[u_{r}, v_{n-r}\right]$ is an outer-commutator of weight $n$. Of course, there exist more than one outer-commutator word of a particular weight $n$.

Now we go back to the groups of matrices. In $U T_{n}(K)$ we distinguish subgroups

$$
U T_{n}^{l}(K)=\left\{\mathbf{1}_{\mathbf{n}}+\sum_{1 \leq i<j-l \leq n-l} a_{i, j} e_{i, j} \mid a_{i, j} \in K\right\}, \quad 0 \leq l \leq n-1 .
$$


The matrix

$$
t_{i, j}(a)=\mathbf{1}_{n}+a e_{i, j} \in G L_{n}(K), \quad i \neq j
$$

is called transvection. It is well known that the set of transvections

$$
\left\{t_{i, j}(a) \mid 1 \leq i<j-l \leq n-l, a \in K\right\}
$$

for every $l \in\{0,1, \ldots, n-1\}$ generates the subgroup $U T_{n}^{l}$ (see [4] for details). Moreover, for an arbitrary field $K$, the descending series of subgroups

$$
U T_{n}(K)=U T_{n}^{0}(K)>U T_{n}^{1}(K)>\ldots>U T_{n}^{n-2}(K)>U T_{n}^{n-1}(K)=\left\{\mathbf{1}_{\mathbf{n}}\right\} .
$$

is the lower central series of $U T_{n}(K)$ with $U T_{n}^{l}(K)=\gamma_{l+1}\left(U T_{n}(K)\right)$.

We recall the concept of 1-generated-as-fully-characteristic subgroups and say that a fully characteristic subgroup $H \leq G$ is 1-generated if there exists an element $g \in H$ such that $H=\langle\varphi(g) \mid \varphi \in \operatorname{End}(G)\rangle$. The following theorem will be proved in Section 2:

Theorem 1 Let $K$ be a field. Every fully characteristic subgroup of the group $\left.U T_{n}(K)\right), n \in \mathbf{N}, n>1$, coincides with a term of the lower central series of this group and is 1-generated as the fully characteristic subgroup.

In other words the first part of Theorem 1 states that for every fully characteristic subgroup $H \leq U T_{n}(K)$ there exists $l \in\{0,1, \ldots, n-1\}$ such that

$$
H=\gamma_{l+1}\left(U T_{n}(K)\right)=U T_{n}^{l}(K) .
$$

From Theorem 1 it follows that

- Every fully characteristic subgroup of $U T_{n}(K)$ is a verbal subgroup in $U T_{n}(K)$.

Since every verbal subgroup coincides with a term of the lower central series in $U T_{n}(K)$, a natural question arising here is: With which term of the lower central series does a given verbal subgroup coincide? For example, we have a well known fact about the derived series of $U T_{n}(K)$ :

- For every $k>0$ we have $V_{d_{k}}\left(U T_{n}(K)\right)=\gamma_{2^{k}}\left(U T_{n}(K)\right)$.

In Section 3 we prove the equalities of another series of verbal subgroups in $U T_{n}(K)$. Namely, we obtain the following result:

Theorem 2 Let $K$ be an arbitrary field and $k$ an integer. The following equalities hold:

1. if char $K=p$ and $k=r \cdot p^{\alpha}$, where $p \nmid r, r, \alpha \in \mathbb{Z}$ and $\alpha \geq 0$, then $V_{x^{k}}\left(U T_{n}(K)\right)=\gamma_{p^{\alpha}}\left(U T_{n}(K)\right)$,

2. if $\omega_{k}$ is an outer-commutator word of weight $k, k>0$, then

$$
V_{\omega_{k}}\left(U T_{n}(K)\right)=\gamma_{k}\left(U T_{n}(K)\right)
$$


The second statement is true for a field of an arbitrary characteristic. It also covers and agrees with the mentioned equalities on verbal subgroups generated by the words $d_{k}$.

Section 4 addresses the problem of verbal width. Due to recent significant applications the width of verbal subgroups in groups has returned to the interest of researchers [3]. Many authors considered the width of verbal subgroups regarding its estimates for certain types of groups or finding examples of groups with finite and infinite verbal width. For example, in [7] it is proved that every verbal subgroup in an algebraic group of matrices over a field $K$, where $K$ is an algebraically closed field of infinite transcendence degree, has finite width. In particular, this result applies to $U T_{n}(K)$ and $T_{n}(K)$. However, there is relatively few results on the exact verbal width in groups. In the following theorem we give the exact width of verbal subgroups of $U T_{n}(K)$ generated by certain words.

Theorem 3 Let $K$ be an arbitrary field and $k \in \mathbf{Z}$.

1. If char $K=p$ then the width wid $_{x^{k}}\left(U T_{n}(K)\right)$ is equal to

(a) 2, if $k=p^{\alpha} \cdot r$ for some nonzero integers $\alpha$ and $r$, such that $\alpha \geq 1$, $p \nmid r$ and $n \geq p^{\alpha}+3 ;$

(b) 1, otherwise.

2. If $\omega_{k}$ is an outer-commutator word of weight $k, k>0$, then $w_{i d_{\omega_{k}}}\left(U T_{n}(K)\right)=1$.

Note that the second statement in Theorem 3 is true regardless of the characteristic of field $K$. By Theorem 3 we get an example of a subgroup having different verbal width, depending on the generating set of words. Moreover, it provides a series of verbal subgroups with finite width greater than 1 .

In Section 5 we characterize the verbal subgroups in the group $T_{n}(K)$. We obtain the following result:

Theorem 4 If $K$ is a field of more than two elements then every verbal subgroup $V_{W}\left(T_{n}(K)\right)$ in the group $T_{n}(K)$ is either $U T_{n}^{l}(K), l \geq 0$ or a product of the form $V_{W}\left(D_{n}(K)\right) \cdot U T_{n}(K)$.

An analogous result for the case of matrix group over the field of characteristic 0 is derived in [1], however the positive characteristic has a great influence on the complexity of the verbal structure. In the last part of the paper we discuss also the case of the groups of infinitely dimensional triangular, unitriangular and diagonal matrices, denoted here as $U T(K), T(K)$ and $D(K)$, respectively. They are defined as the limits of direct systems of groups. Namely, if $\varphi_{n}: T_{n}(K) \hookrightarrow T_{n+1}(K), n \in \mathbf{N}$, are the natural embeddings, such that for all $A \in T_{n}(K)$

$$
\varphi_{n}(A)=\left(\begin{array}{cc}
A & \mathbf{0}^{T} \\
\mathbf{0} & 1
\end{array}\right)
$$


where $\mathbf{0}$ is a zero vector from $K^{n}$, then obviously $\varphi_{n}\left(U T_{n}(K)\right) \subseteq U T_{n+1}(K)$ and $\varphi_{n}\left(D_{n}(K)\right) \subseteq D_{n+1}(K)$. Now, we define:

$$
\begin{aligned}
& T(K)=\underset{n}{\lim }\left(T_{n}(K), \varphi_{n}\right), \\
& U T(K)=\underset{n}{\longrightarrow}\left(U T_{n}(K), \varphi_{n}\right), \\
& D(K)=\underset{n}{\lim }\left(D_{n}(K), \varphi_{n}\right),
\end{aligned}
$$

which are related to each other as follows:

$$
T(K)=D(K) \wedge U T(K) .
$$

Now, the considerations focus on verbal subgroups and their width in the introduced groups of infinitely dimensional matrices. The following result, analogous to the one in [1] for the case of the matrix groups over fields of characteristic 0 , is derived from the direct limit properties:

Theorem 5 Let $K$ be a field.

1. Every verbal subgroup of the group $U T(K)$ coincides with a term of the lower central series of this group and the following equalities hold:

(i) if $\operatorname{char} K=p$ and $p \nmid k$, then wid $_{x^{k}}(U T(K))=1$,

(ii) if char $K=p$ and $k=p^{\alpha} \cdot r$ for some nonzero integers $\alpha$ and $r$, such that $\alpha \geq 1, p \nmid r$ then wid $_{x^{p^{\alpha}}}(U T(K))=2$,

(iii) wid $_{\omega_{k}}(U T(K))=1$ for every outer-commutator word $\omega_{k}$ of weight $k, k \in \mathbf{N}$.

2. Every verbal subgroup $V_{W}(T(K))$ is either a verbal subgroup of $U T(K)$ or can be represented as $V_{W}(D(K)) \cdot U T(K)$.

\section{FULLY CHARACTERISTIC SUBGROUPS OF $U T_{n}(K)$.}

\subsection{Certain endomorphisms of $U T_{n}(K)$.}

In order to describe all fully characteristic subgroups of $U T_{n}(K)$ we first consider its auto- and endomorphisms. There are few classes of automorphisms that can be distinguished in $U T_{n}(K)$, namely the classes of inner and diagonal automorphisms. The second class contains automorphisms of the type $\Delta_{D}$ : $U T_{n}(K) \longrightarrow U T_{n}(K)$, defined as $\Delta_{D}(A)=D^{-1} A D$ where $D \in D_{n}(K)$ is a diagonal matrix. There are also automorphisms of $U T_{n}(K)$ that do not belong to any of these classes, for example $\tau: A \mapsto(\varsigma(A))^{-1}$, where $\varsigma$ is the symmetry with respect to the second diagonal of the matrix.

We introduce two endomorphisms $\beta, \delta: U T_{n}(K) \longrightarrow U T_{n}(K)$ :

$$
\beta\left(\left(\begin{array}{cc}
A & \mathbf{a}^{T} \\
\mathbf{0} & 1
\end{array}\right)\right)=\left(\begin{array}{cc}
1 & \mathbf{0} \\
\mathbf{0}^{T} & A
\end{array}\right),
$$




$$
\delta\left(\left(\begin{array}{cc}
1 & \mathbf{a} \\
\mathbf{0}^{T} & A
\end{array}\right)\right)=\left(\begin{array}{cc}
A & \mathbf{0}^{T} \\
\mathbf{0} & 1
\end{array}\right)
$$

where $\mathbf{a}=\left(a_{1}, a_{2}, \ldots, a_{n-1}\right) \in K^{n-1}, \mathbf{0}=(0,0, \ldots, 0) \in K^{n-1}$ and $A$ is a matrix from $U T_{n-1}(K)$.

Subgroups that are invariant under the automorphisms and endomorphisms described above shall have special form, determined by the way these morphisms act. The latter was studied by Weir and Levchuk, who described all characteristic subgroups in groups of unitriangular matrices over fields $K,|K|>2$ (see $[5,6,9])$.

In the following part of the paper we introduce some specific notation. For every non-identity matrix $A=\left(A_{i, j}\right) \in U T_{n}(K)$ by $d(A)$ we denote the number

$$
d(A)=\min _{1 \leq i<n}\left\{s \mid a_{i, i+s+1} \neq 0\right\} .
$$

Of course, $0 \leq d(A) \leq n-2$. For an arbitrary nontrivial subgroup $H \leq U T_{n}(K)$ we define

$$
D(H)=\min _{A \in H, A \neq \mathbf{1}_{n}} d(A),
$$

which satisfies $0 \leq D(H) \leq n-2$. Obviously $H \subseteq U T_{n}^{D(H)}(K)$.

One of the interesting problems on group morphisms and the characteristic subgroups is the question whether a fully characteristic subgroup is 1-generated. In the following deduction we show that every fully characteristic subgroup in $U T_{n}(K)$ is 1-generated.

\subsection{Proof of Theorem 1 .}

Assume that $H \leq U T_{n}(K)$ is a fully characteristic subgroup of $U T_{n}(K)$. The case of $H=\left\{\mathbf{1}_{n}\right\}$ is trivial, so assume $H \neq\left\{\mathbf{1}_{n}\right\}$. Then it is possible to pick a matrix $A=\left(A_{i, j}\right) \neq \mathbf{1}_{n}$ in $H$ and an index $i_{0} \in\{1,2, \ldots, n-1\}$ in such a way that

$$
A_{i_{0}, i_{0}+D(H)+1}=a \neq 0 .
$$

Since $H$ is fully characteristic subgroup, then every endomorphic image of $A$ is contained in $H$, thus in particular we have:

$$
\delta^{n-D(H)-2}\left(\beta^{n-i_{0}-D(H)-1}(A)\right)=\mathbf{1}_{n}+a e_{1,2+D(H)}=t_{1,2+D(H)}(a) \in H .
$$

Now, take $b \neq 0$ and denote by $D(b)$ the diagonal matrix $\mathbf{1}_{n}+\left(b^{-1}-1\right) e_{1,1}$. Then applying the diagonal automorphism we obtain

$$
\Delta_{D(b)}\left(t_{1,2+D(H)}(a)\right)=t_{1,2+D(H)}(a b) \in H
$$

and hence taking $b=a^{-1} c$ we have $t_{1,2+D(H)}(c) \in H$ for every $c \in K \backslash\{0\}$. Now, applying repeatedly the endomorphism $\beta$ for every $i$ with $1 \leq i \leq n-D(H)-2$ we have

$$
\beta^{i}\left(t_{1,2+D(H)}(c)\right)=t_{i+1, i+2+D(H)}(c) \in H .
$$

A well known fact on commutators of transvections [4] implies that

$$
\left[t_{i, k}(c), t_{k, j}(1)\right]=t_{i, j}(c)
$$


and hence $H$ as a subgroup containing transvections $t_{i, i+D(H)+1}(c)$ for $1 \leq$ $i \leq n-D(H)-1$, contains also all transvections $t_{i, j}(c)$ for $c \in K \backslash\{0\}$, $1 \leq i \leq n-D(H)-1$ and $i+D(H)+1 \leq j \leq n$. These transvections generate the subgroup

$$
U T_{n}^{D(H)}(K)=\gamma_{D(H)+1}\left(U T_{n}(K)\right)
$$

and since the inclusion $H \subseteq U T_{n}^{D(H)}(K)$ is obvious by definition, we have $H=U T_{n}^{D(H)}(K)$.

It is also clear, that $H$ as a fully characteristic subgroup is generated by a single matrix $A$, chosen as indicated in the proof.

\section{VALUES OF POWER AND COMMUTATOR WORDS IN $U T_{n}(K)$ and PROOF OF THEOREM 2}

Theorem 1 states that every verbal subgroup in the group $U T_{n}(K)$ coincides with one of the subgroups $U T_{n}^{l}(K)(0 \leq l \leq n-1)$, which are verbal subgroups generated by the basic commutators $c_{l+1}$. Our goal here is to establish the equalities of verbal subgroups generated by other words and the terms of the lower central series in $U T_{n}(K)$. Namely, two kinds of words will be taken into consideration: the outer-commutator words and the "power" words $x^{k}$. Without loss of generality further we may assume that $k>0$.

\subsection{Values of power words in $U T_{n}(K)$.}

Throughout this section, given a matrix $A \in U T_{n}(K)$, we denote by $\tilde{A}=$ $\left(\tilde{A}_{i j}\right)$ the matrix $A-\mathbf{1}_{\mathbf{n}}$. For every matrix $A$ from $U T_{n}(K)$ and an arbitrary integer $m$ the equality holds:

$$
A^{m}=\sum_{i=0}^{m}\left(\begin{array}{c}
m \\
i
\end{array}\right) \tilde{A}^{i} .
$$

We assume here $\tilde{A}^{0}=\mathbf{1}_{\mathbf{n}}$. The statement is a well known fact for unitriangular matrices over the commutative ring. A simple observation is that $\left(\tilde{A}^{2}\right)_{i, i+1}=0$ for all $1 \leq i<n$ and for $m>2$ the equalities

$$
\left(\tilde{A}^{m}\right)_{i, i+k}=0, \quad 0<k<m
$$

follow easily from induction on $m$. Also, for every $1 \leq i<j \leq n$ we have:

$$
\begin{aligned}
\left(\tilde{A}^{m}\right)_{i, j} & =\sum_{t_{1}=i+1}^{j-1}\left(\tilde{A}^{m-1}\right)_{i, t_{1}} \tilde{A}_{t_{1}, j}=\sum_{t_{1}=i+1}^{j-1} \sum_{t_{2}=i+1}^{t_{1}-1}\left(\tilde{A}^{m-2}\right)_{i, t_{2}} \tilde{A}_{t_{2}, t_{1}} \tilde{A}_{t_{1}, j}= \\
& =\sum_{t_{1}=i+1}^{j-1} \sum_{t_{2}=i+1}^{t_{1}-1} \ldots \sum_{t_{m-1}=i+1}^{t_{m-2}-1} \tilde{A}_{i, t_{m-1}} \tilde{A}_{t_{m-1}, t_{m-2}} \ldots \tilde{A}_{t_{2}, t_{1}} \tilde{A}_{t_{1}, j}= \\
& =\sum_{t_{1}=i+1}^{j-1} \sum_{t_{2}=i+1}^{t_{1}-1} \ldots \sum_{t_{m-1}=i+1}^{t_{m-2}-1} A_{i, t_{m-1}} A_{t_{m-1}, t_{m-2}} \ldots A_{t_{2}, t_{1}} A_{t_{1}, j}
\end{aligned}
$$


and in particular

$$
\begin{aligned}
\left(\tilde{A}^{m}\right)_{i, i+m} & =\sum_{s=i+1}^{i+m-1}\left(\tilde{A}^{m-1}\right)_{i, s} \tilde{A}_{s, i+m}=\left(\tilde{A}^{m-1}\right)_{i, i+m-1} \tilde{A}_{i+m-1, i+m}= \\
& =\left(\tilde{A}^{m-2}\right)_{i, i+m-2} \tilde{A}_{i+m-2, i+m-1} \tilde{A}_{i+m-1, i+m}=\ldots= \\
& =\tilde{A}_{i, i+1} \tilde{A}_{i+1, i+2} \ldots \tilde{A}_{i+m-2, i+m-1} \tilde{A}_{i+m-1, i+m} .
\end{aligned}
$$

The equality (2) also implies the following

Lemma 1 If char $K=p$ and $m=p^{\alpha}, \alpha>0$, then for every matrix $A \in$ $U T_{n}(K)$ we have

$$
A^{m} \in U T_{n}^{m-1}(K)
$$

Proof. Since $p \mid m$ then from Kummer's theorem we have $\left(\begin{array}{c}m \\ i\end{array}\right)=0(\bmod p)$ for $0<i<m$. Hence it follows from (2) that

$$
A^{m}=\mathbf{1}_{\mathbf{n}}+\tilde{A}^{m} .
$$

From (3) it follows immediately that $A^{m} \in U T_{n}^{m-1}(K)$.

\subsection{Values of outer-commutator words in $U T_{n}(K)$.}

We continue with discussion on some basic facts of the outer-commutator words. The considerations below apply for groups of unitriangular matrices over the fields of arbitrary characteristic.

Lemma 2 The matrix $C \in U T_{m+l+2}^{m+l+1}(K)$ is a commutator $C=[A, B]$ of matrices $A \in U T_{m+l+2}^{m}(K)$ and $B \in U T_{m+l+2}^{l}(K)$, such that

$$
\operatorname{rank}(\tilde{A})=l+1, \quad \operatorname{rank}(\tilde{B})=m+1 .
$$

Proof. Let $C \in U T_{m+l+2}^{m+l+1}(K)$. Then $C=\mathbf{1}_{m+l+2}$ and we may choose the matrices $A$ and $B$ as follows:

$$
A=\mathbf{1}_{m+l+2}+\sum_{i=1}^{l+1} e_{i, i+m+1}, \quad B=\mathbf{1}_{m+l+2}+\sum_{i=1}^{m+1} e_{i, i+l+1} .
$$

Then

$$
[A, B]=\mathbf{1}_{m+l+2}=C, \quad \operatorname{rank}(\tilde{A})=l+1, \quad \operatorname{rank}(\tilde{B})=m+1 .
$$

Lemma 3 Let matrix $C \in U T_{n}^{m+l+1}(K)$ be a commutator $C=[A, B]$ of matrices $A \in U T_{n}^{m}(K)$ and $B \in U T_{n}^{l}(K)$, such that

$$
\operatorname{rank}(\tilde{A})=n-m-1, \quad \operatorname{rank}(\tilde{B})=n-l-1 .
$$

Then the matrix

$$
\bar{C}=\left(\begin{array}{ll}
C & \mathbf{c} \\
\mathbf{0} & 1
\end{array}\right)
$$


where $\mathbf{c}^{T}=\left(c_{1}, c_{2}, \ldots, c_{n}\right) \in K^{n}$ and $\mathbf{0}$ is a zero vector from $K^{n}$, is a commutator of matrices $\bar{A} \in U T_{n+1}^{m}(K)$ and $\bar{B} \in U T_{n+1}^{l}(K)$ such that

$$
\bar{A}=\left(\begin{array}{cc}
A & \mathbf{a} \\
\mathbf{0} & 1
\end{array}\right) \quad \bar{B}=\left(\begin{array}{cc}
B & \mathbf{b} \\
\mathbf{0} & 1
\end{array}\right)
$$

where $\mathbf{a}^{T}=\left(a_{1}, a_{2}, \ldots, a_{n}\right), \mathbf{b}^{T}=\left(b_{1}, b_{2}, \ldots, b_{n}\right) \in K^{n}$ and

$$
\operatorname{rank}(\tilde{\bar{A}})=n-m, \quad \operatorname{rank}(\tilde{\bar{B}})=n-l,
$$

where $\tilde{\bar{A}}=\bar{A}-\mathbf{1}_{n+1}$ and $\tilde{\bar{B}}=\bar{B}-\mathbf{1}_{n+1}$.

Proof. Let $\bar{A}$ and $\bar{B}$ be defined as in the statement of the lemma. Then their commutator is a matrix of the form:

$$
\begin{gathered}
{[\bar{A}, \bar{B}]=\left(\begin{array}{cc}
{[A, B]} & \mathbf{x} \\
\mathbf{0} & 1
\end{array}\right)} \\
\mathbf{x}=A^{-1}\left(B^{-1}-\mathbf{1}_{\mathbf{n}}\right) \cdot \mathbf{a}+A^{-1} B^{-1}\left(A-\mathbf{1}_{\mathbf{n}}\right) \cdot \mathbf{b} .
\end{gathered}
$$

For our proof we need to show the existence of vectors $\mathbf{a}$ and $\mathbf{b}$ such that $\mathbf{x}=\mathbf{c}$, $\operatorname{rank}(\tilde{\bar{A}})=n-m$ and $\operatorname{rank}(\tilde{\bar{B}})=n-l$. Since $\bar{C} \in U T_{n+1}^{m+l+1}(K)$ then $\mathbf{c}^{T}=$ $\left(c_{1}, c_{2}, \ldots, c_{n-m-l-1}, 0, \ldots, 0\right)$. Let $D=A^{-1}\left(B^{-1}-\mathbf{1}_{\mathbf{n}}\right)$ and $E=A^{-1} B^{-1}\left(A-\mathbf{1}_{\mathbf{n}}\right)$ and let us choose vectors $\mathbf{d}$ and $\mathbf{e}$ such that

$$
\begin{gathered}
\mathbf{c}=\mathbf{d}+\mathbf{e} \\
d_{n-m-l-1} \neq 0, \quad e_{n-m-l-1} \neq 0, \\
d_{i}=e_{i}=0 \quad \text { for } \quad i>n-m-l-1 .
\end{gathered}
$$

If $c_{n-m-l-1}=0$ then it is a sum of two nonzero opposite elements in $K$, hence there always exist vectors $\mathbf{d}$ and e satisfying the above conditions. Now, the equality $\mathbf{x}=\mathbf{c}$ follows from:

$$
\begin{aligned}
& D \mathbf{a}=\mathbf{d}, \\
& E \mathbf{b}=\mathbf{e},
\end{aligned}
$$

which may be considered as systems of linear equations. Since $\operatorname{rank}(\tilde{A})=$ $n-m-1$ and $A \in U T_{n}^{m}(K)$ then

$$
0 \neq A_{i, i+m+1}=\tilde{A}_{i, i+m+1} \quad \text { for all } \quad i \leq n-m-1 .
$$

Since $\operatorname{rank}(\tilde{B})=n-l-1$ and $B \in U T_{n}^{l}(K)$ then

$$
0 \neq B_{i, i+l+1} \quad \text { for all } \quad i \leq n-l-1
$$

and thus

$$
0 \neq B_{i, i+l+1}^{-1}=\tilde{B}^{-1}{ }_{i, i+l+1} \quad \text { for all } \quad i \leq n-l-1 .
$$

Therefore we have:

$$
\operatorname{rank}(D)=\operatorname{rank}\left(\tilde{B^{-1}}\right)=n-l-1=\operatorname{rank}(D \mid \mathbf{d}),
$$




$$
\operatorname{rank}(E)=\operatorname{rank}(\tilde{A})=n-m-1=\operatorname{rank}(E \mid \mathbf{e}),
$$

where $(D \mid \mathbf{d})$ and $(E \mid \mathbf{e})$ denote the augmented matrices. Hence there exist solutions $\mathbf{a}$ and $\mathbf{b}$ to the systems (7) and (8) respectively. Moreover, as $d_{i}=0$ for $i>n-m-l-1$ we have

$$
a_{n}=a_{n-1}=\ldots=a_{n-m+1}=0
$$

thus $\bar{A} \in U T_{n+1}^{m}(K)$ and since $d_{n-l-m-1} \neq 0$ then $0 \neq a_{n-m}=\bar{A}_{n-m, n+1}=$ $\tilde{\bar{A}}_{n-m, n+1}$ and hence $\operatorname{rank}(\tilde{\bar{A}})=n-m$. Analogically we obtain a solution vector $\mathbf{b}^{T}=\left(b_{1}, \ldots, b_{n-l}, 0, \ldots, 0\right)$, where $b_{n-l} \neq 0$, thus $\bar{B} \in U T_{n+1}^{l}(K)$ and $\operatorname{rank}(\tilde{\bar{B}})=n-l$.

Now, we can combine the two lemmas into one proposition:

Proposition 1 Every matrix $C \in U T_{n}^{m+l+1}(K)$ is a value of a commutator of matrices $A \in U T_{n}^{m}(K)$ and $B \in U T_{n}^{l}(K)$.

Proof. The proof is inductive on the matrix size $n$. Having chosen $m$ and $l$ it is necessary to check the case of the smallest nontrivial group which is for $n=m+l+2$. The checking is stated in Lemma 2 and the inductive step and proof is provided by Lemma 3 .

Proposition 2 Let $\omega_{k}$ be an outer-commutator word of weight $k$. Then

$$
V_{\omega_{k}}\left(U T_{n}(K)\right)=V_{c_{k}}\left(U T_{n}(K)\right) \quad \text { and } \quad w_{i \omega_{\omega_{k}}}\left(U T_{n}(K)\right)=1 .
$$

Proof. The proof is inductive. For $k=1$ we have $\omega_{1}=c_{1}$ thus obviously $V_{\omega_{1}}\left(U T_{n}(K)\right)=V_{c_{1}}\left(U T_{n}(K)\right)$ and $w_{i d_{\omega_{1}}}\left(U T_{n}(K)\right)=1$. Let us assume that the statement is true for all outer-commutator words of weight not greater than $k$. Consider an outer-commutator word $\omega_{k+1}$ of weight $k+1$. There exist outercommutator words $u$ and $v$ of weight $r$ and $k+1-r$, respectively, such that $\omega_{k+1}=[u, v]$. Thus

$$
\begin{aligned}
V_{\omega_{k+1}}\left(U T_{n}(K)\right) & =\left\langle\left[u\left(A_{1}, \ldots, A_{r}\right), v\left(A_{r+1}, \ldots, A_{k+1}\right)\right] \mid A_{i} \in U T_{n}(K)\right\rangle \subseteq \\
& \subseteq\left[V_{u}\left(U T_{n}(K)\right), V_{v}\left(U T_{n}(K)\right)\right]=\left[U T_{n}^{r-1}(K), U T_{n}^{k-r}(K)\right]= \\
& =U T_{n}^{k}(K)=V_{c_{k+1}}\left(U T_{n}(K)\right) .
\end{aligned}
$$

Now we will show the opposite inclusion. We take $C \in V_{c_{k+1}}\left(U T_{n}(K)\right)=$ $U T_{n}^{k}(K)$. Then by Proposition 1 we can choose matrices $A \in U T_{n}^{r-1}(K)$ and $B \in U T_{n}^{k-r}(K)$ such that $C=[A, B]$. From our inductive assumption we have

$$
\begin{array}{ll}
V_{u}\left(U T_{n}(K)\right)=U T_{n}^{r-1}(K), & \operatorname{wid}_{u}\left(U T_{n}(K)\right)=1, \\
V_{v}\left(U T_{n}(K)\right)=U T_{n}^{k-r}(K), & \operatorname{wid}_{v}\left(U T_{n}(K)\right)=1,
\end{array}
$$

hence it follows that there exist matrices $A_{1}, A_{2}, \ldots, A_{k+1} \in U T_{n}(K)$ such that $A=u\left(A_{1}, \ldots, A_{r}\right)$ and $B=v\left(A_{r+1}, \ldots, A_{k+1}\right)$. Thus

$$
C=[A, B]=\left[u\left(A_{1}, \ldots, A_{r}\right), v\left(A_{r+1}, \ldots, A_{k+1}\right)\right]=\omega_{k+1}\left(A_{1}, \ldots, A_{k+1}\right) .
$$

Then $C \in V_{\omega_{k+1}}\left(U T_{n}(K)\right)$ is a value of word $\omega_{k+1}$ in group $U T_{n}(K)$ and hence

$$
V_{\omega_{k+1}}\left(U T_{n}(K)\right)=V_{c_{k+1}}\left(U T_{n}(K)\right) \text { and } \operatorname{wid}_{\omega_{k+1}}\left(U T_{n}(K)\right)=1 \text {. }
$$




\subsection{Proof of Theorem 2.}

We will prove each equality separately.

1. Let us assume that $\operatorname{char} K=p$. Then $U T_{n}(K)$ is a $p$-group and for every integer $r$ such that $p \nmid r$ we have $V_{x^{r}}\left(U T_{n}(K)\right)=U T_{n}(K)$ and $w_{i d} d_{x^{r}}\left(U T_{n}(K)\right)=1$. Thus

$$
V_{x^{r \cdot p^{\alpha}}}\left(U T_{n}(K)\right)=V_{x^{p^{\alpha}}}\left(U T_{n}(K)\right)
$$

and

$$
w_{i d} x_{x^{r \cdot p^{\alpha}}}\left(U T_{n}(K)\right)=w_{i d} x_{p^{\alpha}}\left(U T_{n}(K)\right)
$$

hence it is enough to consider the verbal subgroups generated by power words $x^{p^{\alpha}}$. Following Theorem 1, we need to prove the equality

$$
V_{x^{p^{\alpha}}}\left(U T_{n}(K)\right)=U T_{n}^{p^{\alpha}-1}(K) .
$$

The inclusion $V_{x^{p^{\alpha}}}\left(U T_{n}(K)\right) \subseteq U T_{n}^{p^{\alpha}-1}(K)$ follows Lemma 1, so we need only to prove the reverse inclusion. In the case $\alpha=0$ the equality holds trivially, so further we assume that $\alpha$ is positive. We show that $V_{x^{p^{\alpha}}}\left(U T_{n}(K)\right)$ contains all transvections of the type

$$
t_{i, j}(a)=\mathbf{1}_{\mathbf{n}}+a e_{i j}, \quad a \in K, \quad 1 \leq i \leq n-p^{\alpha}, \quad i+p^{\alpha} \leq j \leq n .
$$

which generate $U T_{n}^{p^{\alpha}-1}(K)$.

Let $m=p^{\alpha}$ and $A \in U T_{n}(K)$. Following (6) we have $A^{m}=\mathbf{1}_{\mathbf{n}}+\tilde{A}^{m}$ and thus by (5)

$$
A_{i, i+m}^{m}=A_{i, i+1} A_{i+1, i+2} \ldots A_{i+m-2, i+m-1} A_{i+m-1, i+m} .
$$

The matrix

$$
B_{i}=\mathbf{1}_{\mathbf{n}}+a e_{i, i+1}+e_{i+1, i+2}+\ldots+e_{i+m-1, i+m}
$$

with $1 \leq i \leq n-m$ satisfies $\left(B_{i}\right)^{m}=t_{i, i+m}(a)$. This argument and the commutator relations

$$
\left[t_{i, i+m}(a), t_{i+m, j}(1)\right]=t_{i, j}(a)
$$

for $i+m<j \leq n$ show that the generators of the group $U T_{n}^{p^{\alpha}-1}(K)$ are contained in $V_{x^{p^{\alpha}}}\left(U T_{n}(K)\right)$, and so the reverse inclusion holds.

2. Let $\omega_{k}$ be an outer-commutator word of weight $k$. The equality

$$
V_{\omega_{k}}\left(U T_{n}(K)\right)=V_{c_{k}}\left(U T_{n}(K)\right)
$$

is a statement of Proposition 2, which has already been proved. 


\section{THE WIDTH OF VERBAL SUBGROUPS.}

In this section we consider the width of verbal subgroups generated by either the words of the form $x^{p^{\alpha}}, \alpha \in \mathbf{N}$ or outer-commutator words. We start with a few simple observations on powers of unitriangular matrices.

Remark 1. Let $\bar{A}$ be a matrix from $U T_{n+1}(K)$, such that

$$
\bar{A}=\left(\begin{array}{ll}
A & \mathbf{a} \\
\mathbf{0} & 1
\end{array}\right)
$$

where $A \in U T_{n}(K), \mathbf{a}^{T}=\left(a_{1}, a_{2}, \ldots, a_{n}\right) \in K^{n}$ and $\mathbf{0}$ is a zero vector from $K^{n}$. Then for any $k \in \mathbf{N}$

$$
\bar{A}^{k}=\left(\begin{array}{cc}
A^{k} & D_{A} \mathbf{a} \\
\mathbf{0} & 1
\end{array}\right),
$$

where

$$
D_{A}=\mathbf{1}_{\mathbf{n}}+A+A^{2}+\ldots+A^{k-1} .
$$

This can be checked by straightforward calculations of $\bar{A}^{k}$.

Remark 2. Let $A$ be a matrix from $U T_{n}(K)$ where $\operatorname{char} K=p$. Then from the equality (5) for every natural number $\alpha$ we have:

$$
A_{n-p^{\alpha}, n}^{p^{\alpha}}=A_{n-p^{\alpha}, n-p^{\alpha}+1} \cdot A_{n-p^{\alpha}+1, n-p^{\alpha}+2} \cdot \ldots \cdot A_{n-1, n}
$$

Remark 3. Let $A$ be a matrix from $U T_{n}(K)$ and let $D=D_{A}$ be defined by (10) for some positive integer $k$. We also use the notation of $\tilde{A}$ from Section 3 . Then, for all $1 \leq i<j \leq n$ by equalities (2) and (4) we have

$$
\begin{aligned}
D_{i, j}= & \sum_{m=1}^{k-1}\left(A^{m}\right)_{i, j}=\sum_{m=1}^{k-1} \sum_{s=0}^{m}\left(\begin{array}{c}
m \\
s
\end{array}\right)\left(\tilde{A}^{s}\right)_{i, j}=\sum_{m=1}^{k-1} \sum_{s=1}^{m}\left(\begin{array}{c}
m \\
s
\end{array}\right)\left(\tilde{A}^{s}\right)_{i, j}= \\
= & \left(\tilde{A}^{1}\right)_{i, j}\left[\left(\begin{array}{c}
1 \\
1
\end{array}\right)+\left(\begin{array}{c}
2 \\
1
\end{array}\right)+\ldots+\left(\begin{array}{c}
k-1 \\
1
\end{array}\right)\right]+ \\
& +\left(\tilde{A}^{2}\right)_{i, j}\left[\left(\begin{array}{l}
2 \\
2
\end{array}\right)+\left(\begin{array}{c}
3 \\
2
\end{array}\right)+\ldots+\left(\begin{array}{c}
k-1 \\
2
\end{array}\right)\right]+\ldots+\left(\tilde{A}^{k-1}\right)_{i, j}\left(\begin{array}{c}
k-1 \\
k-1
\end{array}\right)= \\
= & \sum_{s=1}^{k-1}\left(\sum_{r=s}^{k-1}\left(\begin{array}{c}
r \\
s
\end{array}\right)\left(\tilde{A}^{s}\right)_{i, j}\right)=\sum_{s=1}^{k-1}\left(\begin{array}{c}
k \\
s+1
\end{array}\right)\left(\tilde{A}^{s}\right)_{i, j}= \\
= & \sum_{s=1}^{k-1}\left(\begin{array}{c}
k \\
s+1
\end{array}\right) \sum_{t_{1}=i+1}^{j-1} \sum_{t_{2}=i+1}^{t_{1}-1} \ldots \sum_{t_{s-1}=i+1}^{t_{s-2}-1} A_{i, t_{1}} A_{t_{1}, t_{2}} \ldots A_{t_{s-1}, j}
\end{aligned}
$$

Remark 4. Let $A$ be a matrix from $U T_{n}(K)$ where $\operatorname{char} K=p$ and let $D=$ $D_{A}$ be defined as in (10) for $k=p^{\alpha}$ where $\alpha \in \mathbf{N}$. Then $\left(\begin{array}{l}k \\ s\end{array}\right)=0(\bmod p)$ for $1 \leq s<k$, thus as a consequence of (12) we have $D_{i, j}=0$ in case of $j<i+k-1$ and otherwise

$$
D_{i, j}=\sum_{t_{1}=i+1}^{j-1} \sum_{t_{2}=i+1}^{t_{1}-1} \ldots \sum_{t_{k-2}=i+1}^{t_{k-3}-1} A_{i, t_{1}} A_{t_{1}, t_{2}} \ldots A_{t_{k-2}, j}
$$


In particular

$$
D_{i, i+k-1}=A_{i, i+1} A_{i+1, i+2} \ldots A_{i+k-2, i+k-1}
$$

If $A_{i, i+1} \neq 0$ for $i=1,2, \ldots, n-1$ then $D_{i, i+k-1} \neq 0$ and

$$
\operatorname{rank}(D)=n-k+1
$$

\section{Proof of Theorem 3}

We first prove the first statement of the theorem. Assume that char $K=$ $p>0$ and consider a power word $x^{k}, k \in \mathbb{Z}$. If $k$ is not divisible by $p$ then wid $_{x^{k}}\left(U T_{n}(K)\right)=1$ by arguments given in the proof of Theorem 2 . So assume that $k=r \cdot p^{\alpha}$, where $\alpha \geq 1$. We first check, that if $n \leq p^{\alpha}+2$ then $w_{i d} x_{x^{k}}\left(U T_{n}(K)\right)=1$. Again, by arguments in the proof of Theorem 2, it is enough to show that $w_{i d} x_{p^{\alpha}}\left(U T_{n}(K)\right)=1$. In the following we use the notation of matrix $D_{A}$ defined in (10).

First, note that for $n \leq p^{\alpha}$ we have $V_{x^{p^{\alpha}}}\left(U T_{n}(K)\right)=\left\{\mathbf{1}_{n}\right\}$ and hence obviously $w_{i d} d_{x^{p^{\alpha}}}\left(U T_{n}(K)\right)=1$. Moreover, for $n=p^{\alpha}$ we may choose a matrix $A \in U T_{n}(K)$ such that $A^{p^{\alpha}}=\mathbf{1}_{n}$ and $A_{i, i+1} \neq 0$ for $1 \leq i<n$, and hence by (15) $\operatorname{rank}\left(D_{A}\right)=1$.

Now, assume $n=p^{\alpha}+1$. Then $V_{x^{p^{\alpha}}}\left(U T_{n}(K)\right)=V_{x^{p^{\alpha}}}\left(U T_{p^{\alpha}+1}(K)\right) \neq$ $\left\{\mathbf{1}_{p^{\alpha}+1}\right\}$, so take

$$
B=t_{1, p^{\alpha}+1}(b)=\left(\begin{array}{cc}
\mathbf{1}_{p^{\alpha}} & \mathbf{b} \\
\mathbf{0} & 1
\end{array}\right) \in U T_{p^{\alpha}+1}^{p^{\alpha}-1}(K), \quad \mathbf{b}^{T}=(b, 0,0, \ldots, 0) \in K^{p^{\alpha}},
$$

and denote

$$
\bar{A}=\left(\begin{array}{cc}
A & \mathbf{a} \\
\mathbf{0} & 1
\end{array}\right) \in U T_{p^{\alpha}+1}(K)
$$

where $\mathbf{a}^{T}=\left(a_{1}, a_{2}, \ldots, a_{p^{\alpha}}\right), \mathbf{0} \in K^{p^{\alpha}}$ and $A \in U T_{p^{\alpha}}(K)$ is chosen as above. By (9) we have that $B=\bar{A}^{p^{\alpha}}$ whenever

$$
\mathbf{b}=D_{A} \mathbf{a}
$$

Let $\left(D_{A} \mid \mathbf{b}\right)$ denote the augmented matrix. Due to the properties of $D_{A}$ given in Remark 4 we have

$$
\operatorname{rank}\left(D_{A}\right)=1=\operatorname{rank}\left(D_{A} \mid \mathbf{b}\right) .
$$

By Kronecker-Capelli theorem there exists a solution to the system of linear equations (16) and hence $B$ is a $p^{\alpha}$-th power of $\bar{A}$.

Finally, assume that $n=p^{\alpha}+2$ and take

$$
\bar{B}=\left(\begin{array}{cc}
B & \mathbf{c} \\
\mathbf{0} & 1
\end{array}\right) \in U T_{p^{\alpha}+2}^{p^{\alpha}-1}(K), \quad \mathbf{c}^{T}=\left(c_{1}, c_{2}, 0, \ldots, 0\right) \in K^{p^{\alpha}+1}
$$

Let

$$
\overline{\bar{A}}=\left(\begin{array}{cc}
\bar{A} & \mathbf{d} \\
\mathbf{0} & 1
\end{array}\right) \in U T_{p^{\alpha}+2}(K), \quad \mathbf{d}^{T} \in K^{p^{\alpha}+1}
$$


where $\bar{A}$ is the matrix constructed as in the above reasoning. Note, that in the solution a to the system (16) we obtain $0 \neq a_{p^{\alpha}}=\bar{A}_{p^{\alpha}, p^{\alpha}+1}$ and thus $\operatorname{rank}\left(D_{\bar{A}}\right)=2$. Again, by $(9)$, for $\bar{B}=\overline{\bar{A}}^{p^{\alpha}}$ it is necessary and sufficient that $D_{\bar{A}} \mathbf{d}=\mathbf{c}$. Since $\operatorname{rank}\left(D_{\bar{A}}\right)=2=\operatorname{rank}\left(D_{\bar{A}} \mid \mathbf{c}\right)$ it follows that this equation has a solution. Hence for every matrix $\bar{B} \in U T_{p^{\alpha}+2}^{p^{\alpha}-1}(K)$ there exists $\overline{\bar{A}} \in U T_{p^{\alpha}+2}(K)$ such that $\bar{B}=\overline{\bar{A}}^{p^{\alpha}}$. This completes the proof of part (b) in statement 1 .

The proof of part (a) will consist of two steps. At first we will show that in every group $V_{x^{p^{\alpha}}}\left(U T_{n}(K)\right)$ such that $n \geq p^{\alpha}+3$ with $\alpha \geq 1$ there exists an element which is not a value of the word $x^{p^{\alpha}}$ in group $U T_{n}(K)$. Then we prove that every such element can be represented as a product of two values of the word $x^{p^{\alpha}}$.

We first prove that for every $n \geq p^{\alpha}+3$ and $\alpha \in \mathbf{N}$ we have

$$
\operatorname{wid}_{x^{p^{\alpha}}}\left(U T_{n}(K)\right)>1 \text {. }
$$

Indeed. Assume that for certain $n \geq p^{\alpha}+1$ we have a matrix $B \in V_{x^{p^{\alpha}}}\left(U T_{n}(K)\right)=$ $U T_{n}^{p^{\alpha}-1}(K)$ such that $B_{n-p^{\alpha}, n} \neq 0$ (it is possible to choose such $B$ since $\left.n \geq p^{\alpha}+1\right)$ and there exists $A \in U T_{n}(K)$ satisfying $A^{p^{\alpha}}=B$. Since from (11) we have

$$
A_{n-p^{\alpha}, n}^{p^{\alpha}}=A_{n-p^{\alpha}, n-p^{\alpha}+1} \cdot A_{n-p^{\alpha}+1, n-p^{\alpha}+2} \cdot \ldots \cdot A_{n-1, n}=B_{n-p^{\alpha}, n} \neq 0
$$

then for all $i=n-p^{\alpha}, \ldots, n-1$ we have $A_{i, i+1} \neq 0$. Consider matrix $\overline{\bar{B}} \in$ $U T_{n+2}^{p^{\alpha}-1}(K)$ such that

$$
\overline{\bar{B}}=\left(\begin{array}{ccc}
B & \mathbf{0}^{\mathbf{T}} & \mathbf{1}^{\mathbf{T}} \\
\mathbf{0} & 1 & 0 \\
\mathbf{0} & 0 & 1
\end{array}\right),
$$

where $\mathbf{0}$ is a zero vector form $K^{n}$ and $\mathbf{1} \in K^{n}$ has ones in $n-p^{\alpha}+2$ first coordinates and zeros elsewhere. We denote

$$
\bar{B}=\left(\begin{array}{cc}
B & \mathbf{0}^{\mathbf{T}} \\
\mathbf{0} & 1
\end{array}\right) \in U T_{n+1}^{p^{\alpha}-1}(K) .
$$

If there exists $\bar{A} \in U T_{n+1}(K)$ such that $\bar{B}=\bar{A}^{p^{\alpha}}$ then, by (9), we have

$$
\bar{A}=\left(\begin{array}{cc}
A & \mathbf{a}^{T} \\
\mathbf{0} & 1
\end{array}\right) \quad \text { and } \quad D_{A} \mathbf{a}^{T}=\mathbf{0}^{T}
$$

But

$$
\begin{aligned}
0 & =\bar{B}_{n-p^{\alpha}+1, n+1}=\bar{A}_{n-p^{\alpha}+1, n+1}^{p^{\alpha}}= \\
& =\bar{A}_{n-p^{\alpha}+1, n-p^{\alpha}+2} \cdot \bar{A}_{n-p^{\alpha}+2, n-p^{\alpha}+3} \cdot \ldots \cdot \bar{A}_{n, n+1},
\end{aligned}
$$

where $\bar{A}_{i, j}=A_{i, j}$ for $1 \leq i, j \leq n$. Hence, by assumptions on $A_{i, i+1}$ the solution exists if $\bar{A}_{n, n+1}=0$.

Now, consider $\overline{\bar{B}}$. If $\overline{\bar{B}}=\overline{\bar{A}}^{p^{\alpha}}$, where

$$
\overline{\bar{A}}=\left(\begin{array}{cc}
\bar{A} & \overline{\mathbf{a}}^{T} \\
\overline{\mathbf{0}} & 1
\end{array}\right), \quad D_{\bar{A}} \overline{\mathbf{a}}^{T}=\overline{\mathbf{1}}^{T}
$$


and $\overline{\mathbf{a}}=\left(\mathbf{a}, a_{n+1}\right) \in K^{n+1}$ and $\overline{\mathbf{1}}=(\mathbf{1}, 0) \in K^{n+1}$, then we have

$$
\begin{aligned}
1 & =\overline{\bar{B}}_{n-p^{\alpha}+2, n+2}=\overline{\bar{A}}_{n-p^{\alpha}+2, n+2}^{p^{\alpha}}= \\
& =\overline{\bar{A}}_{n-p^{\alpha}+2, n-p^{\alpha}+3} \cdot \overline{\bar{A}}_{n-p^{\alpha}+3, n-p^{\alpha}+4} \cdot \ldots \cdot \overline{\bar{A}}_{n, n+1} \cdot \overline{\bar{A}}_{n+1, n+2}
\end{aligned}
$$

But $\overline{\bar{A}}_{n, n+1}=\bar{A}_{n, n+1}=0$, hence there is no solution to the above equation and thus $\overline{\bar{B}}$ is not a value of the word $x^{p^{\alpha}}$ in group $U T_{n+2}(K)$.

Now, we will show that every element from $V_{x^{p^{\alpha}}}\left(U T_{n}(K)\right)$ is a product of two values of the word $x^{p^{\alpha}}$ in group $U T_{n}(K)$. The proof will be inductive with respect to the matrix dimension $n$. First, we observe from Theorem 2 that for small dimensions $n$ the verbal subgroups generated by the words $x^{p^{\alpha}}$ are trivial. Hence the one and only matrix from such verbal subgroup is $\mathbf{1}_{\mathbf{n}}$ and can be represented as a product of $p^{\alpha}$-powers of any two matrices from $U T_{n}(K)$. In particular, it is true that for every matrix $B \in V_{x^{p^{\alpha}}}\left(U T_{n}(K)\right)$ we can choose matrices $A, C \in U T_{n}(K)$ such that:

$$
B=A^{p^{\alpha}} C^{p^{\alpha}}, \quad A_{i, i+1} C_{i, i+1} \neq 0
$$

for every $i$ in $\{1, \ldots, n-1\}$.

Now for the inductive proof assume that, for certain dimension $n$, every matrix $B$ from the verbal subgroup $V_{x^{p^{\alpha}}}\left(U T_{n}(K)\right)$ can be represented as a product of powers of $A, C \in U T_{n}(K)$, satisfying (17). We take $\bar{B} \in U T_{n+1}(K)$ and, using our inductive assumptions we have:

$$
\bar{B}=\left(\begin{array}{cc}
B & \mathbf{b} \\
\mathbf{0} & 1
\end{array}\right)=\left(\begin{array}{cc}
A^{p^{\alpha}} C^{p^{\alpha}} & \mathbf{b} \\
\mathbf{0} & 1
\end{array}\right)
$$

where $A, B, C \in U T_{n}(K)$ and $\mathbf{b}$ is a transposed vector from $K^{n}$.

Let us denote

$$
\bar{A}=\left(\begin{array}{cc}
A & \mathbf{a} \\
\mathbf{0} & 1
\end{array}\right), \quad \bar{C}=\left(\begin{array}{cc}
C & \mathbf{c} \\
\mathbf{0} & 1
\end{array}\right),
$$

for some vectors $\mathbf{a}^{T}, \mathbf{c}^{T} \in K^{n}$. Then we have

$$
\overline{A^{p^{\alpha}}} \overline{C^{p^{\alpha}}}=\left(\begin{array}{cc}
A^{p^{\alpha}} C^{p^{\alpha}} & A^{p^{\alpha}} D_{C} \cdot \mathbf{c}+D_{A} \cdot \mathbf{a} \\
\mathbf{0} & 1
\end{array}\right)
$$

where $D_{A}$ and $D_{C}$ are defined by (10).

It is clear that $D_{A}$ and $D_{C}$ are not invertible. By (15) we have that $\operatorname{rank}\left(D_{A}\right)=\operatorname{rank}\left(D_{C}\right)$ is equal to $n-p^{\alpha}+1$ as $A$ and $C$ satisfy (17). For the equality $\bar{B}=\bar{A}^{\alpha} C^{p^{\alpha}}$ it is necessary that

$$
A^{p^{\alpha}} D_{C} \cdot \mathbf{c}+D_{A} \cdot \mathbf{a}=\mathbf{b}
$$

Since $\bar{B} \in V_{x^{p^{\alpha}}}\left(U T_{n+1}(K)\right)=U T_{n+1}^{p^{\alpha}-1}(K)$ then

$$
\mathbf{b}=\left(b_{1}, b_{2}, \ldots, b_{n-p^{\alpha}+1}, 0, \ldots, 0\right) .
$$

Now, we can represent the vector $\mathbf{b}$ as a sum of two vectors $\mathbf{e}$ and $\mathbf{f}$ from $K^{n}$

$$
\mathbf{b}=\mathbf{e}+\mathbf{f}
$$


such that $f_{n-p^{\alpha}+1} \neq 0$ and $e_{n-p^{\alpha}+1} \neq 0$ and $e_{i}=f_{i}=0$ for $i>n-p^{\alpha}+1$. We have

$$
\begin{aligned}
\operatorname{rank}\left(D_{A}\right) & =\operatorname{rank}\left(D_{A} \mid \mathbf{f}\right) \\
\operatorname{rank}\left(A^{p^{\alpha}} D_{C}\right) & =\operatorname{rank}\left(A^{p^{\alpha}} D_{C} \mid \mathbf{e}\right),
\end{aligned}
$$

and hence there exist solutions $\mathbf{x}=\mathbf{c}$ and $\mathbf{y}=\mathbf{a}$ to the systems of linear equations

$$
A^{p^{\alpha}} D_{C} \cdot \mathbf{x}=\mathbf{e}, \quad D_{A} \cdot \mathbf{y}=\mathbf{f} .
$$

Moreover, if $D_{A} \mathbf{a}=\mathbf{f}$ then

$$
\bar{A}^{p^{\alpha}}=\left(\begin{array}{cc}
A^{p^{\alpha}} & \mathbf{f} \\
\mathbf{0} & 1
\end{array}\right)
$$

and since from (11) we get $0 \neq f_{n-p^{\alpha}+1}=\bar{A}_{n-p^{\alpha}+1, n+1}^{p^{\alpha}}=\prod_{i=n-p^{\alpha}+1}^{n} \bar{A}_{i, i+1}$ thus $\bar{A}_{n, n+1} \neq 0$ and by (14) $\operatorname{rank}\left(D_{\bar{A}}\right)=n-k+2$. In addition, as $A^{p^{\alpha}}$ is invertible and triangular, then there exist unique solution $\mathbf{u}$ to the equation:

$$
A^{p^{\alpha}} \mathbf{u}=\mathbf{e}
$$

such that $u_{n-p^{\alpha}+1}=e_{n-p^{\alpha}+1} \neq 0$ and $u_{i}=0$ for $i \geq n-p^{\alpha}+2$. Then, by the same arguments as above, we obtain that $\bar{C}_{n, n+1} \neq 0$ and $\operatorname{rank}\left(D_{\bar{C}}\right)=n-k+2$. Hence $\bar{B}=\bar{A}^{p^{\alpha}} \bar{C}^{p^{\alpha}}$, where $\bar{A}_{i, i+1} \bar{C}_{i, i+1} \neq 0$ for all $i=1,2, \ldots, n$. Thus, by induction, wid $_{x^{p^{\alpha}}}\left(U T_{n}(K)\right)=2$.

The second part of Theorem 3 has been stated and proved as Proposition 3 in subsection 3.2. It should be pointed out that the case of $\omega_{k}$ being a right-normed basic commutator and the field being of characteristic 0 has been studied and stated as Theorem 4 in the earlier work of the author [1]. The proof of Theorem 4 was based on the precedent Lemma 5, which is true but proved incorrectly. The misstatement was noticed and corrected by the author in [2]. Also the detailed proof of Proposition 2 presented above covers the case and completes the calculations and proof of Theorem 4 in [1].

\section{PROOFS OF THEOREMS 4 AND 5}

\subsection{Verbal subgroups in $T_{n}(K)$}

The characterization of verbal subgroups in $U T_{n}(K)$ enables further considerations on verbal structure in the group of triangular matrices $T_{n}(K)$. Since the group $T_{n}(K)$ is a semidirect product of the subgroup $D_{n}(K)$ and the normal subgroup $U T_{n}(K)$, every verbal subgroup of $T_{n}(K)$ must be of the form $V_{W}\left(T_{n}(K)\right)=V_{W}\left(D_{n}(K)\right) H$ for some subgroup $H$ of $U T_{n}(K)$, normal in $T_{n}(K)$ and including the verbal subgroup $V_{W}\left(U T_{n}(K)\right)$.

Now we consider the verbal subgroups generated by various types of words separately. 
1. Verbal subgroups generated by commutator words

If $w$ is a commutator word then $V_{w}\left(D_{n}(K)\right)=\left\{\mathbf{1}_{\mathbf{n}}\right\}$ and $V_{w}\left(T_{n}(K)\right)=$ $H \subseteq U T_{n}(K)$ and moreover, $H$ is a fully characteristic subgroup of $T_{n}(K)$. We extend the endomorphisms $\beta$ and $\delta$ of $U T_{n}(K)$ to the endomorphisms $\bar{\beta}$ and $\bar{\delta}$ of $T_{n}(K)$ in the following way:

$$
\bar{\beta}\left(\left(\begin{array}{cc}
A & \mathbf{a}^{T} \\
\mathbf{0} & \alpha
\end{array}\right)\right)=\left(\begin{array}{cc}
1 & \mathbf{0} \\
\mathbf{0}^{T} & A
\end{array}\right), \quad \bar{\delta}\left(\left(\begin{array}{cc}
\alpha & \mathbf{a} \\
\mathbf{0}^{T} & A
\end{array}\right)\right)=\left(\begin{array}{cc}
A & \mathbf{0}^{T} \\
\mathbf{0} & 1
\end{array}\right) .
$$

Arguing as in the proof of Theorem 1 we deduce that $H=U T_{n}^{m}(K)$, where $m=D(H)$.

2. Verbal subgroups generated by power words

Let us assume that $K$ is a finite field consisting of $q$ elements and $\operatorname{char} K=$ $p, q>2$. The multiplicative group $K^{*}$ of field $K$ is cyclic. Let $a$ be the generator of $K^{*}$. If $r$ is a natural number such that $0<r<q-1$, then $a^{r} \neq 1$. In particular, for every $k<q-1$ the equality

$$
a^{k}-1=(a-1)\left(1+a+a^{2}+\ldots+a^{k-1}\right)
$$

implies

$$
1+a+a^{2}+\ldots+a^{k-1}=\left(a^{k}-1\right)(a-1)^{-1} .
$$

We consider the following cases :

(a) $(q-1) \mid m$

Then the equality $y^{q-1}=1$ satisfied in the group $K^{*}$ implies that $D^{m}=\mathbf{1}_{\mathbf{n}}$ for every matrix $D \in D_{n}(K)$, i.e. $V_{x^{m}}\left(D_{n}(K)\right)=\left\{\mathbf{1}_{\mathbf{n}}\right\}$. Hence $V_{x^{m}}\left(T_{n}(K)\right) \subseteq U T_{n}(K)$, and since $V_{x^{m}}\left(T_{n}(K)\right)$ is fully characteristic, it is invariant to endomorphisms $\bar{\beta}$ and $\bar{\delta}$ and thus it must be equal to $U T_{n}^{l}(K)$ for $l=D\left(V_{x^{m}}\left(T_{n}(K)\right)\right)$.

(b) $(q-1) \nmid m$

Then $a^{m} \neq 1$. If $m<q-1$ then $a^{m} \neq 1$ because $a$ is the generator of the cyclic group $K^{*}$. If $m>q-1$, then there exist numbers $s, t \in \mathbf{Z}$, such that $m=(q-1) \cdot s+t$ and $t<q-1$. Hence

$$
a^{m}=a^{(q-1) \cdot s+t}=\left(a^{q-1}\right)^{s} \cdot a^{t}=a^{t} \neq 1 .
$$

For $i=1,2, \ldots, n-1$ and arbitrary $b \in K$ we define the triangular matrix

$$
A_{i}=\mathbf{1}_{\mathbf{n}}+(a-1) \cdot e_{i, i}+b \cdot e_{i, i+1} .
$$

Then the equality (19) implies that

$$
\begin{aligned}
\left(A_{i}\right)^{m} & =\mathbf{1}_{\mathbf{n}}+\left(a^{m}-1\right) e_{i, i}+\left(b \sum_{j=0}^{m-1} a^{j}\right) e_{i, i+1}= \\
& =\mathbf{1}_{\mathbf{n}}+\left(a^{m}-1\right) e_{i, i}+b\left(a^{m}-1\right)(a-1)^{-1} e_{i, i+1} .
\end{aligned}
$$


Let $D_{i}$ be a diagonal matrix:

$$
D_{i}=\mathbf{1}_{\mathbf{n}}+e_{i, i} \cdot\left(a^{-1}-1\right)
$$

then

$$
\left(D_{i}\right)^{m}=\mathbf{1}_{\mathbf{n}}+e_{i, i} \cdot\left(a^{-m}-1\right)
$$

and

$$
\begin{aligned}
\left(D_{i}\right)^{m} \cdot\left(A_{i}\right)^{m} & =\mathbf{1}_{\mathbf{n}}+b\left(a^{m}-1\right)(a-1)^{-1} a^{-m} e_{i, i+1}= \\
& =\mathbf{1}_{\mathbf{n}}+b(a-1)^{-1}\left(1-a^{-m}\right) e_{i, i+1} \in V_{x^{m}}\left(T_{n}(K)\right) .
\end{aligned}
$$

Now, we observe that for every $c \in K$ there exists $b \in K$ such that

$$
b(a-1)^{-1}\left(1-a^{-m}\right)=c .
$$

Indeed. Since $a^{m} \neq 1$ then $a^{-m} \neq 1$ and $\left(1-a^{-m}\right)$ is an invertible element. Then one can take

$$
b=c \cdot\left(1-a^{-m}\right)^{-1}(a-1) .
$$

The verbal subgroup $V_{x^{m}}\left(T_{n}(K)\right)$ contains the set of all unitriangular matrices of the form:

$$
\mathbf{1}_{\mathbf{n}}+c \cdot e_{i, i+1}, \quad c \in K,
$$

which generates the whole group $U T_{n}(K)$. Hence $U T_{n}(K) \subseteq V_{x^{m}}\left(T_{n}(K)\right)$ and finally we have

$$
V_{x^{m}}\left(T_{n}(K)\right)=V_{x^{m}}\left(D_{n}(K)\right) \cdot U T_{n}(K) .
$$

Now, we summarize the above observation in the proof of Theorem 4 .

Proof of Theorem 4. Let us consider first the verbal subgroup $V_{w}\left(T_{n}(K)\right)$ generated by a single word $w$. If $K$ is a finite field consisting of $q$ elements, $q>2$, then - as shown above - if $w$ is either a commutator word or a noncommutator word in which all letterwise sums of exponents are divisible by $q-1$ then the verbal subgroup $V_{w}\left(T_{n}(K)\right)$ coincides with $U T_{n}^{m}(K)$ for certain $m \in \mathbf{N}$. Otherwise $w$ is a non-commutator word containing a letter with the sum of exponents not divisible by $q-1$ and generates the verbal subgroup of the form $V_{w}\left(D_{n}(K)\right) \cdot U T_{n}(K)$.

Since $T_{n}(K)$ is not nilpotent, then we need to consider the verbal subgroups generated by the sets of words $W=\left\{w_{i}, i \in I\right\}$. By definition

$$
V_{W}\left(T_{n}(K)\right)=\prod_{i \in I} V_{w_{i}}\left(T_{n}(K)\right),
$$

and every subgroup $V_{w_{i}}\left(T_{n}(K)\right)$ is either of the form $(1): V_{w_{i}}\left(D_{n}(K)\right) \cdot U T_{n}(K)$ or of the form (2): $U T_{n}^{m}(K), m \geq 0$. If $W$ contains at least one word generating the verbal subgroup of the form (1), then the verbal subgroup $V_{w}\left(T_{n}(K)\right)$ contains $U T_{n}(K)$ and all possible values of commutator words. Therefore

$$
V_{W}\left(T_{n}(K)\right)=\prod_{i \in I} V_{w_{i}}\left(T_{n}(K)\right)=\prod_{i \in I^{\prime}} V_{w_{i}}\left(D_{n}(K)\right) \cdot U T_{n}(K)=V_{W}\left(D_{n}(K)\right) \cdot U T_{n}(K),
$$


where $I^{\prime}$ is the set of indices, for which $w_{i}$ generates the verbal subgroup of the form (1).

Otherwise, every word $w_{i} \in W$ generates one of the subgroups $U T_{n}^{m_{i}}(K)$. Then

$$
V_{W}\left(T_{n}(K)\right)=\prod_{i \in I} V_{w_{i}}\left(T_{n}(K)\right)=\prod_{i \in I} U T_{n}^{m_{i}}(K)=U T_{n}^{M}(K),
$$

where $M$ is the least number among $m_{i}$. Thus, every verbal subgroup $V_{W}\left(T_{n}(K)\right)$ is of the form stated in the theorem.

\subsection{Proof of Theorem 5}

It is easy to verify (see [1]), that if $\left(G_{n}, \varphi_{n}\right), n \in \mathbf{N}$ is a direct system of groups and $G=\underset{n}{\lim _{n}} G_{n}$ is the limit of this system of groups, then for every set of words $W$ the following equality holds

$$
V_{W}(G)=\underset{n}{\lim } V_{W}\left(G_{i}\right)
$$

This implies statements 1 and 2 of the theorem (without items (i), (ii) and (iii)).

Now, consider a verbal subgroup $V_{W}(U T(K))$ such that wid $_{W}\left(U T_{n}(K)\right)=1$ for every $n \in \mathbf{N}$. Let $A$ be a matrix from $V_{W}(U T(K))$. Then there exists $N \in \mathbb{N}$ such that $A$ represents a matrix $A_{N} \in V_{W}\left(U T_{N}(K)\right)$. Since $\operatorname{wid}_{W}\left(U T_{N}(K)\right)=$ 1 then $A_{N}$ is a value of a word $w$ from $W$ in $U T_{N}(K)$ and there exist matrices $B_{N j} \in U T_{N}(K), 1 \leq j \leq t$ such that $A=w\left(B_{N 1}, \ldots, B_{N t}\right)$. Thus $A$ is a value of the word $w$ on the representatives of $B_{N j}$ in $U T(K)$ and hence statements (i) and (iii) follow easily. In addition, statement (ii) follows Theorem 3 and the precedent reasoning.

ACKNOWLEDGEMENTS. The author would like to thank to the anonymous referee for many useful comments and remarks that helped to improve the overall clarity of the content and for indicating the reference to the related announcement of Yu. V. Sosnovskii [8].

\section{REFERENCES}

[1] Bier A., "Verbal subgroups in the group of triangular matrices over field of characteristic 0", J. Algebra, vol. 321 No .2 (2009), p. 483-494.

[2] Bier, A., "Lattices of verbal subgroups in nilpotent groups"(polish), PhD Thesis, University of Silesia, 2010.

[3] Segal, D., "Words. Notes on verbal width in groups", London Math. Soc. LNS 361, Cambridge 2009

[4] Kargapolov M. I., Merzlyakov Yu. I. "Fundamentals of the theory of groups", Springer, 1979. 
[5] Levchuk V. M., "Subgroups of the unitriangular group", Izv. Ross. Akad. Nauk Ser. Mat., 38 (1974), p.1202-1220.

[6] Levchuk V. M., "Connections between the unitriangular group and certain rings. II. Groups of automorphisms", Sibirsk. Mat. Zh. 24 (1983), No.4, p. 64-80.

[7] Merzlyakov Yu. I. "Algebraic linear groups as complete automorphism groups and completeness of their verbal subgroups", Algebra i Logika 6 (1967), No. 1, p. $83-94$.

[8] Sosnovskii Yu. V., "Verbal subgroups in the groups of triangular and unitriangular matrices over a field of arbirtrary characteristic", International Conference in Memory of A. I. Mal'cev, Novosibirsk, 2009.

[9] Weir A. J., "Sylow $p$-subgroups of the general linear group over finite fields of characteristic p", Proc. Amer. Math. Soc., Vol. 6, No. 3 (1955), p.454-464.

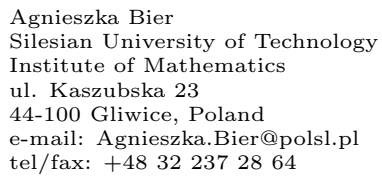

